\title{
On discreteness of spectrum and positivity of the Green's function for a second order functional-differential operator on semiaxis
}

Sergey Labovskiy ${ }^{1 *}$ and Mário Frengue Getimane ${ }^{2}$

${ }^{\text {"Correspondence: }}$ labovski@gmail.com 'Department of Mathematics, Moscow State University of Economics, Statistics and Informatics, Nezhinskaya 7, Moscow, 119501, Russia Full list of author information is available at the end of the article

\begin{abstract}
We study conditions of discreteness of spectrum of the operator defined by $\mathcal{L} u=-\frac{1}{\rho(x)} u^{\prime \prime}(x)-\int_{0}^{\infty} u(s) d_{s} r(x, s), x \in[0, \infty)$. The operator has two singularities at the ends of the interval $(0, \infty)$. The second question is positivity of solutions of the equation $\mathcal{L} u=f$ under boundary conditions $u(0)=0, u^{\prime}(\infty)=0$. The used abstract scheme is close to the well-known MS Birman's method in the spectral theory of self-adjoint operators. Conditions for discreteness of spectrum and positivity of the Green's operator are obtained. The result relates to the MS Birman's result on the necessary and sufficient condition for discreteness of spectrum of a polar-differential operation. The results may be interesting for researchers in qualitative theory of functional-differential equations and spectral theory of self-adjoint operators.
\end{abstract}

MSC: Primary 34K08; 34K10; secondary 34K12

Keywords: discreteness of spectrum; positive solutions; singular operator

\section{Introduction}

\subsection{Problems and a well-known result}

Our first objective is to study the conditions for discreteness of spectrum ${ }^{\mathrm{a}}$ of the functional-differential operator defined by

$$
\mathcal{L} u=-\frac{1}{\rho(x)} u^{\prime \prime}-\int_{0}^{\infty} u(s) d_{s} r(x, s), \quad x \in R_{+}=[0, \infty)
$$

with two singularities: at $x=0$ and at infinity. Note that one particular case of the expression (1.1) is the following operator with one deviation:

$$
-\frac{1}{\rho} u^{\prime \prime}(x)-q(x) u(h(x))
$$

The second question is existence of positive solutions of the equation $\mathcal{L} u=f$ (see Definition 1.1).

Note the result of Birman [1, (Chapter 2, Section 29)] for the spectral problem

$$
\mathcal{L}_{0} u \stackrel{\text { def }}{=}-\frac{1}{\rho} u^{\prime \prime}=\lambda u, \quad x \in R_{+},
$$


where the operation $\mathcal{L}_{0}$ is called polar-differential operation. This singular spectral problem is usually considered in the space $L_{2}\left(R_{+}, \rho\right)$ of functions that are square-integrable on $R_{+}$with the positive weight $\rho$. Birman showed that a necessary and sufficient condition of discreteness of spectrum of the operator $\mathcal{L}_{0}$ is

$$
\lim _{s \rightarrow \infty} s \int_{s}^{\infty} \rho(x) d x=0 .
$$

The singularity at the point $x=0$ is not reflected in this condition. If $\int_{0}^{1} \rho(x) d x=\infty$, condition (1.3) is not sufficient for discreteness. We impose the second condition,

$$
\lim _{s \rightarrow 0} s \int_{s}^{\infty} \rho(x) d x=0
$$

The two conditions (1.3) and (1.4) are sufficient for discreteness of the spectrum of (1.2) (Theorem 2.1). ${ }^{\mathrm{b}}$ It seems that (1.4) is also necessary one.

Part of this work is a continuation of the research in the articles [2-4].

\subsection{Assumptions, notation}

Everywhere below, except for the independent appendix, we use the assumptions and notation introduced in this subsection.

The function $\rho$ is assumed to be measurable and positive almost everywhere on $R_{+}=$ $[0, \infty)$ and satisfying the important condition

$$
\sup _{s \in(0, \infty)} s \int_{s}^{\infty} \rho(x) d x<\infty
$$

Remark 1.1 Comparing (1.3), (1.4), and (1.5) we see that the key role is played by the properties of the function

$$
\Phi(s)=s \int_{s}^{\infty} \rho(x) d x .
$$

Conditions (1.3) and (1.4) can be written as $\lim _{s \rightarrow \infty} \Phi(s)=\lim _{s \rightarrow 0} \Phi(s)=0$, and condition (1.5) is boundedness of the $\Phi(s)$. The latter is sufficient (Lemma 4.3) for the inclusion $W \subset L_{2}\left(R_{+}, \rho\right)$ and for boundedness of the operator $T$ (see, below in this subsection). It is close to a necessary condition: if, for example, $\lim _{s \rightarrow \infty} \Phi(s)=\infty$, then $T$ is unbounded (see Remark 4.3).

Everywhere below the function $r(x, s)$ is assumed to satisfy the following conditions: it is nondecreasing in $s \in R_{+}$for almost all $x \in R_{+}$, it is measurable in $x$ for any $s \in R_{+}$, and

$$
q(x)=r(x, \infty)-r(x, 0)
$$

is locally integrable on $(0, \infty)$. We can assume that $r(x, 0)=0$ for almost all $x \in R_{+}$(as follows from a property of the Stieltjes integral).

Now, let us introduce the function

$$
\xi(x, y)=\rho(x) \int_{0}^{x} r(s, y) d s
$$


Finally, let

- $L_{2}\left(R_{+}, \rho\right)$ be the Hilbert space of all square-integrable with positive almost everywhere weight $\rho$ on $R_{+}=[0, \infty)$ functions $f$, i.e. $\int_{R_{+}} f(x)^{2} \rho(x) d x<\infty$, and with scalar product

$$
(f, g)=\int_{R_{+}} f(x) g(x) \rho(x) d x,
$$

- $W$ be the set of all locally absolutely continuous on $R_{+}$functions $u$ satisfying

$$
\int_{R_{+}} u^{\prime}(x)^{2} d x<\infty
$$

and the boundary condition $u(0)=0$; $W$ is a Hilbert space (Lemma 4.1) with scalar product

$$
[u, v]=\int_{R_{+}} u^{\prime}(x) v^{\prime}(x) d x
$$

and

- $T: W \rightarrow L_{2}\left(R_{+}, \rho\right)$ be the operator defined by $\operatorname{Tu}(x)=u(x), x \in R_{+}$. Note that $W \subset L_{2}\left(R_{+}, \rho\right)$ under condition (1.5) (Lemma 4.3).

\subsection{About domain of the operators $\mathcal{L}_{0}$ and $\mathcal{L}$}

If $\lambda$ is a regular value of $\mathcal{L}_{0}$, then any solution of $-\frac{1}{\rho} u^{\prime \prime}-\lambda u=f$ satisfies $-\frac{1}{\rho} u^{\prime \prime} \in L_{2}\left(R_{+}, \rho\right)$. So, we have to assume that the domain $D\left(\mathcal{L}_{0}\right)$ of the operator $\mathcal{L}_{0}$ consists of all solutions of the equation

$$
-\frac{1}{\rho} u^{\prime \prime}=z \in L_{2}\left(R_{+}, \rho\right) \text {. }
$$

These solutions have locally on $(0, \infty)$ absolutely continuous derivative $u^{\prime}$ and have the form

$$
u(x)=\int_{0}^{\infty} G_{0}(x, s) z(s) d s+u(0)+u^{\prime}(\infty) x,
$$

where $G_{0}(x, s)=\rho(s) \min \{x, s\}$. In fact, since $\left(\int_{x}^{\infty} \rho z d s\right)^{2} \leq \int_{x}^{\infty} \rho z^{2} d s \int_{x}^{\infty} \rho d s$ the integral is convergent, and $u^{\prime}(x)=\int_{x}^{\infty} \rho(t) z(t) d t+u^{\prime}(\infty)$. From this we have

$$
u(x)=\int_{0}^{x} \int_{s}^{\infty} \rho z d t d s+u(0)+u^{\prime}(\infty) x=\int_{0}^{\infty} G_{0}(x, s) z(s) d s+u(0)+u^{\prime}(\infty) x .
$$

We impose the boundary conditions $u(0)=0, u^{\prime}(\infty)=0$. Both of these conditions are necessary for the implementation of the variational method in the space of $W$. Then the domain is determined automatically (see Corollary A.1 to Lemma A.1 and Lemma 4.8).

Definition 1.1 We say that the boundary value problem

$$
\begin{aligned}
& -\frac{1}{\rho} u^{\prime \prime}-\int_{0}^{\infty} u(s) d_{s} r(x, s)=f, \quad f \in L_{2}\left(R_{+}, \rho\right), \\
& u(0)=0, \quad u^{\prime}(\infty)=0
\end{aligned}
$$


is positively solvable, if it is uniquely solvable for any $f \in L_{2}\left(R_{+}, \rho\right)$, and the implication $f \geq 0 \rightarrow u \geq 0$ holds.

Note that positive solvability is equivalent to the positivity of the Green's function $G(x, s)$, which allows one to represent the solution of $\mathcal{L} u=f$ in the form

$$
u(x)=\int_{0}^{\infty} G(x, s) f(s) d s
$$

or $u=G f$, where $G$ is the Green's operator of $\mathcal{L}$.

\section{Discreteness of spectrum}

\subsection{Operator $\mathcal{L}_{0}$}

Instead of direct investigation of the equation $\mathcal{L}_{0} u=f$, where $f \in L_{2}\left(R_{+}, \rho\right)$, consider the equation

$$
\int_{0}^{\infty} u^{\prime}(x) v^{\prime}(x) d x=\int_{0}^{\infty} f(x) v(x) \rho(x) d x, \quad \forall v \in W .
$$

Equation (2.1) is the result of the variational method, in which the primary object is the form $[u, v]$. Equation (2.1) has the short form

$$
[u, v]=(f, T v),
$$

defined on the spaces $W$ and $H=L_{2}\left(R_{+}, \rho\right)$. Note that $W$ is a Hilbert space (Lemma 4.1), $T: W \rightarrow L_{2}\left(R_{+}, \rho\right)$ is bounded (Lemma 4.3), and $T(W)$ is dense in $L_{2}\left(R_{+}, \rho\right)$. So, we can use the abstract scheme in Appendix 1. According to Corollary A.1 (2.1) is equivalent to an equation $\mathcal{L}_{0} u=f$. By virtue of Lemma 4.8 (2.1) is equivalent to

$$
-\frac{1}{\rho} u^{\prime \prime}=f, \quad u(0)=u^{\prime}(\infty)=0 .
$$

Theorem 2.1 Suppose conditions (1.3) and (1.4) hold. Then the spectral problem

$$
-\frac{1}{\rho} u^{\prime \prime}=\lambda u, \quad u(0)=0, \quad u^{\prime}(\infty)=0
$$

has a discrete spectrum.

Proof The discreteness follows from Theorem A.1 and Lemma 4.7.

Remark 2.1 (Estimate of the greatest lower bound of the spectrum) Let $\lambda_{0}$ be the greatest lower bound of spectrum of the operator $\mathcal{L}_{0}$. Thus the problem

$$
-\frac{1}{\rho} u^{\prime \prime}=\lambda u+f, \quad u(0)=0, \quad u(\infty)=0
$$

for $\lambda<\lambda_{0}$ is uniquely resolvable for any $f \in L_{2}\left(R_{+}, \rho\right)$, but it is not if $\lambda=\lambda_{0}$. Then in view of (A.4) from inequality (4.3) it follows that

$$
\frac{1}{\lambda_{0}}=\sup _{u \neq 0} \frac{(T u, T u)}{[u, u]} \leq 4 \sup \left(s \int_{s}^{\infty} \rho(x) d x\right) .
$$


This is an accurate estimate. If $\rho(x)=1 / x^{2}$, then $\lambda_{0} \geq 1 / 4$, but $\lambda=1 / 4$ is a point of the spectrum, as follows from Example 2.1.

Example 2.1 If $\rho(x)=1 /\left(x^{2}\right)$, then $s \int_{s}^{\infty} \rho(s) d s \equiv 1$, and the operator $T$ is bounded, but the two conditions (1.3) and (1.4) are not fulfilled. The spectrum of the operator $\mathcal{L}_{0} u=-x^{2} u^{\prime \prime}$ in $L_{2}\left(R_{+}, \rho\right), \rho=1 /\left(x^{2}\right)$ is the interval $[1 / 4, \infty)$.

In fact, the value $\lambda<1 / 4$ is regular (Remark 2.1). Let $\lambda>1 / 4$.

By means of the change of variable $x=e^{t}$ the equation

$$
-x^{2} u^{\prime \prime}-\lambda u=f
$$

can be transformed to $-u^{\prime \prime}(t)+u^{\prime}(t)-\lambda u=\varphi$, where $\varphi=f\left(e^{t}\right)$. Since

$$
\int_{R_{+}} f(x)^{2} \rho(x) d x=\int_{R} \varphi(t)^{2} e^{-t} d t
$$

this equation has to be considered in the space $L_{2}\left(R, e^{-t}\right)$.

The homogeneous equation $-u^{\prime \prime}+u^{\prime}-\lambda u=0$ has the solution $u=e^{(1 / 2) t}\left(c_{1} \cos \delta t+\right.$ $\left.c_{2} \sin \delta t\right)$ for a $\delta>0$. It is not in $L_{2}\left(R, e^{-t}\right)$. Let $u=e^{(1 / 2) t} \sin \delta t v(t)=b(t) v(t)$. Then

$$
\begin{aligned}
-u^{\prime \prime}+u^{\prime}-\lambda u & =-\left(b^{\prime \prime} v+2 b^{\prime} v^{\prime}+b v^{\prime \prime}\right)+\left(b^{\prime} v+b v^{\prime}\right)-\lambda b v=\left(-2 b^{\prime}+b\right) v^{\prime}-b v^{\prime \prime} \\
& =-2 \delta e^{(1 / 2) t} \cos \delta t v^{\prime}-e^{(1 / 2) t} \sin \delta t v^{\prime \prime}=\varphi .
\end{aligned}
$$

If, for example, $v=1 / \sqrt{t}, t \geq 1$, and $v=0, t<0$ (for $0 \leq t \leq 1$ the $v(t)$ may be defined arbitrarily), then $\varphi \in L_{2}\left(R, e^{-t}\right)$. But the corresponding solution $u \notin L_{2}\left(R, e^{-t}\right)$. Thus, $\lambda$ is not a regular value of the operator.

Since the spectrum is a real closed set, $[1 / 4, \infty)$ is the spectrum.

\subsection{General operator $\mathcal{L}$}

The operator (1.1) can be represented as $\mathcal{L}=\mathcal{L}_{0}-Q$, where $Q$ is defined by

$$
Q u(x)=\int_{0}^{\infty} u(s) d_{s} r(x, s) .
$$

The operator $Q$ acts from $W$ to $L_{2}\left(R_{+}, \rho\right)$ and is bounded under certain conditions (see, for example, (5.6)). Along with the general case, let us consider one special case (deviating operator):

$$
Q u(x)=q(x) u(h(x)),
$$

where $q(x)$ is assumed to be nonnegative locally integrable function, and $h(x)$ is a measurable function. Note that the notation $q(x)$ in (2.5) corresponds to the definition (1.7), if represent expression (2.5) in the form (2.4).

Theorem 2.2 If conditions (1.3) and (1.4) are fulfilled and $Q: W \rightarrow L_{2}\left(R_{+}, \rho\right)$ is bounded, then spectrum of $\mathcal{L}$ is discrete. If the function $\xi$ defined by (1.8) is symmetric, i.e. $\xi(x, y)=$ $\xi(y, x)$ for all $x, y \in R_{+}$, then the spectrum is real and the system of eigenfunctions has the orthogonal basis properties in $L_{2}\left(R_{+}, \rho\right)$. 
Proof Conditions (1.3) and (1.4) are sufficient conditions of compactness of the operator $T$ (Lemma 4.7). The symmetry condition of $\xi(x, y)$ allows one to show the identity $(Q u, T v)=$ $(Q v, T u)$ (see Section 5). Now we can refer to Theorem A.3.

Using the estimate (5.6) from Theorem A.3 we have the first main result.

Theorem 2.3 Suppose (1.3) and (1.4) hold and

$$
\sup _{s} \int_{0}^{\infty} \rho(x) q(x)(q(x)-r(x, s)) d x<\infty .
$$

Then the spectral problem

$$
\begin{aligned}
& -\frac{1}{\rho} u^{\prime \prime}(x)-\int_{0}^{\infty} u(s) d_{s} r(x, s)=\lambda u, \quad x \in(0, \infty), \\
& u(0)=u^{\prime}(\infty)=0
\end{aligned}
$$

has a discrete spectrum. If $\xi(x, y)=\xi(y, x),{ }^{\mathrm{c}}$ the spectrum is real, and the system of eigenfunctions has orthogonal basis properties in the spaces $W$ and $L_{2}\left(R_{+}, \rho\right)$.

Remark 2.2 The spectrum is not real in general, because of the non-symmetry of the function $\xi(x, y)$.

The obtained estimate works well in the case of one deviation, if $Q$ is defined by (2.5). From (2.6) we have the following.

Corollary 2.1 Suppose (1.3) and (1.4) hold and

$$
\sup _{s}\left(s \int_{h(x) \geq s} \rho(x) q(x)^{2} d x\right)<\infty .
$$

Then the spectral problem

$$
\begin{aligned}
& -\frac{1}{\rho} u^{\prime \prime}(x)-q(x) u(h(x))=\lambda u, \quad x \in(0, \infty), \\
& u(0)=u^{\prime}(\infty)=0
\end{aligned}
$$

has a discrete spectrum.

Example 2.2 If $Q u(x)=q(x) u(x)$ the operator $\mathcal{L}$ has the representation

$$
\mathcal{L} u=-\frac{1}{\rho(x)} u^{\prime \prime}-q(x) u .
$$

If (1.3) and (1.4) are satisfied and

$$
\sup _{s} \int_{s}^{\infty} \rho q^{2} d x<\infty
$$

then the operator $\mathcal{L}$ has discrete spectrum. In this case (2.6) has the form (2.8). It follows also from (5.4). Note that the inequality (2.8) is satisfied, if $q(x)$ is bounded. 


\section{Positive solvability}

Suppose $Q: W \rightarrow L_{2}\left(R_{+}, \rho\right)$ is bounded. By the substitution $u=T^{*} z$ the equation $\mathcal{L} u=f$ is reduced to the equation

$$
z-K z=f
$$

where $K=Q T^{*}$ is an integral operator with nonnegative kernel,

$$
K(x, t)=\int_{0}^{\infty} G_{0}(s, t) d_{s} r(x, s) .
$$

Thus, if spectral radius of $K$ is less than unit $(\rho(K)<1)$, then $(3.1)$ is uniquely resolvable and

$$
f \geq 0 \rightarrow z \geq 0 .
$$

Since ${ }^{\mathrm{d}} z=(I-K)^{-1} f$ and $u=G_{0} z$, the operator $G=G_{0}(I-K)^{-1}$ is positive, and $G(x, s) \geq 0$. Thus:

Theorem 3.1 Suppose $Q$ is bounded and $\rho(K)<1$. Then the boundary value problem (1.13), (1.14) is uniquely resolvable for any $f \in L_{2}\left(R_{+}, \rho\right)$ and the Green's operator $G$ is positive.

Remark 3.1 Since $\rho(K) \leq\|K\|=\left\|T^{*} Q\right\| \leq\left\|T^{*}\right\|\|Q\|=\|T\|\|Q\|$ the condition $\|T\|\|Q\|<1$ is sufficient for positivity of the Green's operator $G$.

The second main result is presented in the following statement.

Theorem 3.2 If

$$
\sup _{s}\left(s \int_{s}^{\infty} \rho d x\right) \sup _{s}\left(s \int_{s}^{\infty} \rho(x) q(x)(q(x)-r(x, s)) d x\right)<\frac{1}{16}
$$

then the boundary value problem (1.13), (1.14) is positively solvable (see Definition 1.1).

Proof See Remark 3.1 to Theorem 3.1 and the estimates (4.2) and (5.6).

Consider the following particular case.

\section{Corollary 3.1 The equation}

$$
-\frac{1}{\rho} u^{\prime \prime}(x)-q(x) u(h(x))=f
$$

$(q \geq 0)$ has a positive solution in $W$ for any $f \in L_{2}\left(R_{+}, \rho\right), f \geq 0, f \neq 0$, if

$$
\sup _{s}\left(s \int_{s}^{\infty} \rho d x\right) \sup _{s}\left(s \int_{h(x) \geq s} \rho(x) q(x)^{2} d x\right)<\frac{1}{16} .
$$


The following particular case shows that in this estimate the inequality sign $<$ cannot be replaced by $\leq$.

Example 3.1 From Theorem 3.1 and (5.4) it follows that the equation

$$
-\frac{1}{\rho(x)} u^{\prime \prime}-q(x) u=f
$$

$(q(x) \geq 0)$ is uniquely resolvable for $f \in L_{2}\left(R_{+}, \rho\right)$ in $W$ and has positive solution for $f \geq 0$, if

$$
\sup _{s}\left(s \int_{s}^{\infty} \rho q^{2} d x\right) \cdot \sup _{s}\left(s \int_{s}^{\infty} \rho(x) d x\right)<\frac{1}{16}
$$

In particular, if $q(x) \leq$ const $=q$, and $\rho(x)=1 / x^{2},\left\|T^{*} Q\right\| \leq 16 q^{2}$. Thus, if $4 q<1$, the equation $-x^{2} u^{\prime \prime}-q(x) u=f$ has unique positive solution for any $f \in L_{2}\left(R_{+}, \rho\right), f \geq 0, f \neq 0$.

But if $q(x)=$ const $=1 / 4$, the equation $-x^{2} u^{\prime \prime}-(1 / 4) u=f$ may not have a solution for some $f$ (this was considered in Example 2.1).

In concluding this section consider one useful assertion. Let

$$
\langle u, v\rangle=\int_{R_{+}} u^{\prime} v^{\prime} d x-\int_{R_{+} \times R_{+}} u(s) v(x) d \xi
$$

and

$$
Q(u, v)=\int_{R_{+} \times R_{+}} u(s) v(x) d \xi
$$

Note that $Q(u, v)=(Q u, T v)$ (see the equality (5.2)).

Let $\lambda_{0}$ be the greatest lower bound of spectrum of the operator $\mathcal{L}$. Then by (A.10)

$$
\lambda_{0}=\inf _{u \neq 0} \frac{\langle u, u\rangle}{(T u, T u)}
$$

From Theorem A.5 follows the following.

Theorem 3.3 Suppose conditions (1.3) and (1.4) hold. Then $\lambda_{0}$ is the smallest eigenvalue of the problem $\mathcal{L} u=\lambda u, u(0)=u^{\prime}(+\infty)=0$, and the following statements are equivalent:

1. $\langle u, u\rangle$ is positive definite,

2. $\lambda_{0}>0$,

3. $\rho\left(Q T^{*}\right)<1$.

Remark 3.2 We do not suppose that $\lambda_{0}>-\infty$ (but if $\rho\left(Q T^{*}\right)<1$ it is so).

\section{Auxiliaries propositions}

Recall that in all assertions below condition (1.5) is assumed to be fulfilled.

Lemma 4.1 $W$ is a Hilbert space. 
Proof The relation $u(x)=\int_{0}^{x} z(s) d s$ establishes a bijection between $W$ and the Hilbert space $L_{2}\left(R_{+}\right)$.

Lemma 4.2 The value $A_{u}=\int_{R_{+}} \frac{\left|u(s) u^{\prime}(s)\right|}{s} d s$ satisfies the inequality

$$
A_{u} \leq 2[u, u]
$$

Proof This follows from the inequalities

$$
\begin{aligned}
A_{u}^{2} & \leq \int_{R_{+}} \frac{u^{2}}{s^{2}} d s \int_{R_{+}}\left(u^{\prime}\right)^{2} d s=2 \int_{R_{+}} \frac{d s}{s^{2}} \int_{0}^{s} u(t) u^{\prime}(t) d t[u, u] \\
& =2 \int_{R_{+}} \frac{u(t) u^{\prime}(t)}{t} d t[u, u] \leq 2 A_{u} \cdot[u, u] .
\end{aligned}
$$

Remark 4.1 The estimate (4.1) is accurate, since if $u=s^{1 / 2-\varepsilon}, s \geq 1$, and $u=s^{1 / 2+\varepsilon}, s \geq 1$, then

$$
\frac{2}{1+2 \varepsilon} \leq \frac{A_{u}}{[u, u]}
$$

$\operatorname{Remark} 4.2$ If $u(s) \geq 0, u^{\prime}(s) \geq 0, u^{\prime \prime}(s) \leq 0$, then

$$
A_{u} \geq 0.5[u, u]
$$

In fact, denote $B_{u}=\int_{R_{+}} \frac{u^{2}}{s^{2}} d s$. Then $B_{u}=2 A_{u}$. Since $u(s)=\int_{0}^{s} u^{\prime}(t) d t \geq s u^{\prime}(s), B_{u} \geq$ $\int_{R_{+}} u^{\prime}(s)^{2} d s=[u, u]$.

Lemma 4.3 Suppose (1.5) holds. Then $T(W) \subset L_{2}\left(R_{+}, \rho\right)$, the operator $T: W \rightarrow L_{2}\left(R_{+}, \rho\right)$ is bounded, and the norm of $T$ satisfies the estimate

$$
\|T\|^{2} \leq 4 \sup _{s \in R_{+}}\left(s \int_{s}^{\infty} \rho(x) d x\right)
$$

Proof Since $(T u, T u)=\int_{R_{+}} u^{2} \rho d x=2 \int_{R_{+}} \rho(x) d x \int_{0}^{x} u(s) u^{\prime}(s) d s$, we can estimate

$$
(T u, T u) \leq 2 \int_{R_{+}} \rho(x) d x \int_{0}^{x}\left|u(s) u^{\prime}(s)\right| d s=2 \int_{R_{+}} \frac{\left|u(s) u^{\prime}(s)\right|}{s} s \int_{s}^{\infty} \rho(x) d x d s .
$$

From this and (4.1)

$$
(T u, T u) \leq 4 \sup _{s \in R_{+}}\left(s \int_{s}^{\infty} \rho(x) d x\right)[u, u]
$$

Remark 4.3 If $\lim _{s \rightarrow \infty} \Phi(s)=\infty$ (see (1.6)), then $T(W) \not \subset L_{2}\left(R_{+}, \rho\right)$. In fact, if $u(s) u^{\prime}(s) \geq 0$, then $(T u, T u)=2 \int_{R_{+}} \frac{u(s) u^{\prime}(s)}{s} \Phi(s) d s$. It is possible to find a nonincreasing function $\psi(s)$ such that

$$
\int_{R_{+}} \frac{\psi(s)}{s} d s<\infty, \quad \text { but } \int_{R_{+}} \frac{\psi(s)}{s} \Phi(s) d s=\infty
$$


Now find $u$ such that $u(s) u^{\prime}(s)=\psi(s): u(s)^{2}=2 \int_{0}^{s} \psi(t) d t$. Since $u \geq 0, u^{\prime}(s) \geq 0$, and $u^{\prime}$ is nonincreasing, by Remark 4.2, $[u, u] \leq 2 A_{u}=\int_{R_{+}} \frac{\psi(s)}{s} d s<\infty$. But $(T u, T u)=\infty$.

Lemma 4.4 The image $T(W)$ is dense in $L_{2}\left(R_{+}, \rho\right)$.

Proof The proof is left to the reader.

The following theorem [5, p.318] can be used to show compactness.

Theorem 4.1 (Gelfand) A set E from a separable Banach space $X$ is relatively compact if and only iffor any sequence of linear continuous functionals that converge to zero at each point

$$
f_{n}(x) \rightarrow 0, \quad \forall x \in X
$$

the convergence (4.4) would be uniform on the E.

Lemma 4.5 Suppose (1.3) holds. If $f_{n} \in L_{2}\left(R_{+}, \rho\right)$ is bounded, then

$$
\lim _{N \rightarrow \infty} \int_{N}^{\infty} f_{n}(x) u(x) \rho(x) d x=0
$$

uniformly on the set $\{(u, n):\|u\| \leq 1, n \in\{1,2, \ldots\}\}$.

Proof Since

$$
\left(\int_{N}^{\infty} f_{n}(x) u(x) \rho(x) d x\right)^{2} \leq \int_{N}^{\infty} f_{n}(x)^{2} \rho(x) d x \int_{N}^{\infty} u(x)^{2} \rho(x) d x
$$

it is sufficient to show that

$$
\int_{N}^{\infty} u(x)^{2} \rho(x) d x \rightarrow 0
$$

uniformly on $\|u\| \leq 1$. We have

$$
\int_{N}^{\infty} u^{2} \rho d x=\int_{N}^{\infty} \rho d x\left(u(N)^{2}+2 \int_{N}^{x} u(s) u^{\prime}(s) d s\right) .
$$

The first term tends to zero because of the inequality

$$
u(N)^{2}=\left(\int_{0}^{N} u^{\prime}(s) d s\right)^{2} \leq \int_{0}^{N} u^{\prime}(s)^{2} d s \cdot \int_{0}^{N} 1 d s \leq N
$$

and (1.3). The second term is equal to

$$
2 \int_{N}^{\infty} \rho d x \int_{N}^{x} u(s) u^{\prime}(s) d s=2 \int_{N}^{\infty} \frac{u(s) u^{\prime}(s)}{s}\left(s \int_{s}^{\infty} \rho d x\right) d s
$$

This tends to zero because of (4.1) and (1.3). 
Lemma 4.6 Suppose (1.4) holds. If $f_{n} \in L_{2}\left(R_{+}, \rho\right)$ is bounded, then

$$
\lim _{a \rightarrow 0} \int_{0}^{a} f_{n}(x) u(x) \rho(x) d x=0
$$

uniformly on the set $\{(u, n):\|u\| \leq 1, n \in\{1,2, \ldots\}\}$.

Proof Since

$$
\begin{aligned}
\left(\int_{0}^{a} f_{n}(x) u(x) \rho(x) d x\right)^{2} & \leq \int_{0}^{a} f_{n}(x)^{2} \rho(x) d x \int_{0}^{a} u(x)^{2} \rho(x) d x \\
& \leq C \int_{0}^{a} u(x)^{2} \rho(x) d x
\end{aligned}
$$

it is sufficient to show that

$$
\int_{0}^{a} u(x)^{2} \rho(x) d x \rightarrow 0
$$

when $a \rightarrow 0$ uniformly on $\|u\| \leq 1$. We have

$$
\int_{0}^{a} u^{2} \rho d x=\int_{0}^{a}\left(2 \int_{0}^{x} u(s) u^{\prime}(s) d s\right) \rho d x=2 \int_{0}^{a} \frac{u(s) u^{\prime}(s)}{s}\left(s \int_{s}^{a} \rho(x) d x\right) d s .
$$

Now we refer to (1.4) and (4.1).

Lemma 4.7 If (1.3) and (1.4) hold, then $T$ is compact.

Proof Let $\Omega=\{T u:\|u\| \leq 1\}$. We use the criterium of compactness of Gelfand (see Theorem 4.1). Let $f_{n} \in L_{2}\left(R_{+}, \rho\right)$ be a sequence of functionals such that $f_{n}(z) \rightarrow 0$ for any $z \in L_{2}\left(R_{+}, \rho\right)$. We have to show that $f_{n}(T u) \rightarrow 0$ uniformly for $\|u\| \leq 1$.

Let $\varepsilon>0$. Using Lemma 4.5 choose $N$ such that

$$
\left|\int_{N}^{\infty} f_{n}(x) u(x) \rho(x) d x\right|<\varepsilon / 4
$$

for all $n$ and for all $\|u\| \leq 1$. The same we can do with $\int_{0}^{a} f_{n}(x) u(x) \rho(x) d x$ for sufficiently small $a>0$. For this aim we can use Lemma 4.6.

Now we only have to show that $\int_{a}^{N} f_{n}(x) u(x) \rho(x) d x \rightarrow 0$ uniformly on the set $\|u\| \leq 1$. Since

$$
\begin{aligned}
\left(\int_{a}^{N} f_{n}(x) u(x) \rho(x) d x\right)^{2} & =\left(\int_{a}^{N} f_{n}(x)\left(\int_{a}^{x} u^{\prime}(s) d s\right) \rho(x) d x\right)^{2} \\
& =\left(\int_{a}^{N} u^{\prime}(s) d s \int_{s}^{N} f_{n}(x) \rho(x) d x\right)^{2} \\
& \leq \int_{a}^{N} u^{\prime}(s)^{2} d s \int_{a}^{N} \varphi_{n}(s)^{2} d s \\
& \leq \int_{a}^{N} \varphi_{n}(s)^{2} d s
\end{aligned}
$$


where $\varphi_{n}(s)=\int_{s}^{N} f_{n}(x) \rho(x) d x$, it suffices to show that $\int_{a}^{N} \varphi_{n}(s)^{2} d s \rightarrow 0$. We show that $\varphi_{n}(s) \rightarrow 0$ uniformly for $s \in[a, N]$. Let

$$
z_{s}(x)= \begin{cases}1 & \text { if } x \in[s, N], \\ 0 & \text { if } x \notin[s, N] .\end{cases}
$$

Then $\varphi_{n}(s)=f_{n}\left(z_{s}\right)$. The set $\left\{z_{s}: s \in[a, N]\right\}$ is compact. Thus by Theorem 4.1 of Gelfand $f_{n}\left(z_{s}\right)$ converges to zero uniformly for these $s$. So, $\varphi_{n}(s) \rightarrow 0$ uniformly for $s \in[a, N]$, and $f_{n}(T u) \rightarrow 0$ uniformly for $\|u\| \leq 1$.

Remark 4.4 It seems that condition (1.4) is necessary for compactness of $T$.

Lemma 4.8 Equation (2.1) is equivalent to problem (2.2).

Proof Denote $h^{\prime}=-f \rho$. From (2.1) it follows that

$$
\int_{0}^{\infty} u^{\prime} v^{\prime} d x=-\int_{0}^{\infty} h^{\prime} v d x=-\left.v h\right|_{0} ^{\infty}+\int_{0}^{\infty} h v^{\prime} d x
$$

(if we choose $v$ such that the corresponding limits exist). If $v=0$ for $x \notin[a, b] \subset(0, \infty)$, then $\int_{a}^{b} u^{\prime} v^{\prime} d x=\int_{a}^{b} h v^{\prime} d x$. From this $u^{\prime}=h+$ const on $[a, b]$. Since the segment $[a, b]$ is arbitrary, the relation $u^{\prime}=h+$ const is fulfilled on the whole semiaxis $(0, \infty)$. So, $u^{\prime \prime}=h^{\prime}=$ $-\rho f$. By the first equality in (4.5)

$$
\int_{0}^{\infty} u^{\prime} v^{\prime} d x=-\int_{0}^{\infty} u^{\prime \prime} v d x=-\left.u^{\prime} v\right|_{0} ^{\infty}+\int_{0}^{\infty} u^{\prime} v^{\prime} d x
$$

Now, $\left.u^{\prime} v\right|_{x=\infty}=\left.u^{\prime} v\right|_{x=0}=0$. Choosing $v$ such that $v(\infty) \geq C>0$, we obtain $u^{\prime}(\infty)=0$.

\section{Operator Q. Symmetry and estimates of the norm}

Here we consider the operator $Q$ defined by (2.4).

\subsection{Symmetry of the form $(Q u, T v)$}

Under the assumptions imposed on the functions $r(x, s)$ and $\xi(x, y)$ in Section 1.2, from Lemma B.1 (in Appendix 2) we obtain the following statement.

\section{Lemma 5.1}

$$
\int_{R_{+} \times R_{+}} f(x, y) d \xi=\int_{R_{+}} d x \rho(x) \int_{R_{+}} f(x, y) d_{y} r(x, y) .
$$

In this case $X=Y=R_{+}, \mu(e)=\int_{e} \rho(x) d x, K(x, d y)=r(x, d y)=d_{y} r(x, y)$.

Using Lemma 5.1 the form

$$
(Q u, T v)=\int_{R_{+}} d x v(x) \rho(x) \int_{R_{+}} u(s) d_{s} r(x, s)
$$

can be represented in the symmetrical form

$$
(Q u, T v)=\int_{R_{+} \times R_{+}} u(s) v(x) d \xi .
$$

Hence, this form is symmetric if the function $\xi(x, y)$ is symmetric: $\xi(x, s)=\xi(s, x)$. 


\subsection{Unique deviation}

Consider first the special case when the operator $Q$ is defined by (2.5), i.e. $Q u(x)=$ $q(x) u(h(x))$. Using (4.1) we have

$$
\begin{aligned}
(Q u, Q u) & =\int_{0}^{\infty} \rho(x) q(x)^{2} u(h(x))^{2} d x=\int_{0}^{\infty} d x \rho q^{2} 2 \int_{0}^{h(x)} u(s) u^{\prime}(s) d s d x \\
& =2 \int_{0}^{\infty} \frac{u(s) u^{\prime}(s)}{s}\left(s \int_{h(x) \geq s} \rho(x) q(x)^{2} d x\right) d s \\
& \leq 4[u, u] \sup _{s} s \int_{h(x) \geq s} \rho(x) q(x)^{2} d x .
\end{aligned}
$$

From this follows the estimate of $\|Q\|$ :

$$
\|Q\|^{2} \leq 4 \sup _{s}\left(s \int_{h(x) \geq s} \rho(x) q(x)^{2} d x\right) .
$$

In particular, when $Q u(x)=q(x) u(x)$,

$$
\|Q\|^{2} \leq 4 \sup _{s} \int_{s}^{\infty} \rho q^{2} d x .
$$

\subsection{General operator $Q$}

Here we consider the general case of the operator $Q$ defined by (2.4), i.e. $Q u(x)=$ $\int_{0}^{\infty} u(t) d_{t} r(x, t)$. Suppose that the function $\xi(x, y)=\rho(x) \int_{0}^{x} r(s, y) d s$ (see (1.8)) is absolutely continuous in $y$, and $\xi(x, y)=\int_{0}^{y} p(x, t) d t$. Then $p(x, t)$ does not decrease in $x$.

In this case

$$
\begin{aligned}
(Q u(x))^{2} & =\int_{R_{+}} u(t) d_{t} r(x, t) \int_{R_{+}} u(\tau) d_{\tau} r(x, \tau)=\iint_{R_{+} \times R_{+}} u(t) u(\tau) d_{t} r(x, t) d_{\tau} r(x, \tau) \\
& \leq \frac{1}{2} \iint_{R_{+} \times R_{+}}\left(u(t)^{2}+u(\tau)^{2}\right) d_{t} r(x, t) d_{\tau} r(x, \tau)=\int_{R_{+}} u(t)^{2} d_{t} r(x, t) q(x),
\end{aligned}
$$

where $q(x)$ is defined by (1.7). Using Lemma 5.1, we obtain

$$
\begin{aligned}
\|Q u\|^{2} & =\int_{R_{+}} d x \rho(x)(Q u(x))^{2} \leq \int_{R_{+}} d x \rho(x) q(x) \int_{R_{+}} u(t)^{2} d_{t} r(x, t) \\
& =\int_{R_{+} \times R_{+}} q(x) u(t)^{2} d \xi=\int_{R_{+}} d t u(t)^{2} \int_{R_{+}} q(x) d_{x} p(x, t) \\
& \leq 4[u, u] \sup _{s} \int_{s}^{\infty} d t \int_{R_{+}} q(x) d_{x} p(x, t) .
\end{aligned}
$$

The latter step can be done in the same manner as in the relation (5.4).

From this

$$
\|Q\|^{2} \leq 4 \sup _{s} s \int_{s}^{\infty} d t \int_{R_{+}} q(x) d_{x} p(x, t) .
$$

Since

$$
\int_{s}^{\infty} d t \int_{R_{+}} q(x) d_{x} p(x, t)=\int_{R_{+}} d x \rho(x) q(x) \int_{s}^{\infty} d_{t} r(x, t),
$$


it may be presented in the form

$$
\|Q\|^{2} \leq 4 \sup _{s} \int_{R_{+}} \rho(x) q(x)(q(x)-r(x, s)) d x .
$$

This estimate only works well if for all $x$ deviation is concentrated around the point $h(x)$. For example, if $r(x, s)=q(x) r_{1}(s-h(x)), r_{1}(+\infty)=1, r_{1}(-\infty)=0$ then

$$
\|Q\|^{2} \leq 4 \sup _{s} \int_{R_{+}} \rho(x) q(x)^{2}\left(1-r_{1}(s-h(x))\right) d x .
$$

This estimate coincides with (5.3), if $r_{1}(t)= \begin{cases}0 & \text { if } t \leq 0, \\ 1 & \text { if } t>0\end{cases}$

In this case

$$
\int_{R_{+}} u(s) d_{s} r(x, s)=q(x) u(h(x)) .
$$

\section{Appendix 1: Abstract scheme}

We do not use general spectral theory (see, for example, $[6,7])$. But the scheme below is close to the scheme in [7, Chapter 10] except for using a different notation. We find also convenient explicit use of the embedding $T$ from $W$ to $H$ (see below). This scheme was used also in [2-4].

\section{A.1 Positive form}

Let $W$ and $H$ be Hilbert spaces with inner product $[u, v]$ and $(f, g)$, respectively. Let $T: W \rightarrow H$ be a linear bounded operator. The equation

$$
[u, v]=(f, T v), \quad \forall v \in W,
$$

has the unique solution $u=T^{*} f$ for any $f \in H$, where $T^{*}$ is adjoint operator. Let $D_{\mathcal{L}}=$ $T^{*}(H)$.

Assume that

1. the image $T(W)$ of the operator $T$ is dense in $H$,

2. $\operatorname{dim} \operatorname{ker} T=0$.

Lemma A.1 If the image $T(W)$ of the operator $T$ is dense in $H$, then $T^{*}$ is an injection.

Proof Suppose $T^{*} f=0$ for a $f \in H$. Then for any $g \in T(W)$

$$
(f, g)=(f, T u)=\left[T^{*} f, u\right]=0 .
$$

Since $T(W)$ is dense in $H$, the $f=0$.

Corollary A.1 (Euler equation) The operator $T^{*}$ has an inverse $\mathcal{L}$ defined on the set $D_{\mathcal{L}}$. Equation (A.1) is equivalent to

$$
\mathcal{L} u=f \text {. }
$$


The spectral problem for the operator $\mathcal{L}$ we will write in the form

$$
\mathcal{L} u=\lambda T u .
$$

Let $\lambda_{0}$ be the greatest lower bound of the spectrum of $\mathcal{L}$. It is well known (see, for example, [7, Chapter 6]) that

$$
\lambda_{0}=\inf _{u \neq 0} \frac{(\mathcal{L} u, T u)}{(T u, T u)} .
$$

Since $(\mathcal{L} u, T u)=\left[T^{*} \mathcal{L} u, u\right]=[u, u]$,

$$
\lambda_{0}=\inf _{u \neq 0} \frac{[u, u]}{(T u, T u)}=\|T\|^{-2} .
$$

Theorem A.1 The spectrum of $\mathcal{L}$ is discrete if and only if $T$ is compact.

Proof Since (A.3) is equivalent to $u=\lambda T^{*} T u$, discreteness of spectrum of $\mathcal{L}$ is equivalent to compactness of $T^{*} T$. But the operators $T^{*} T$ and $T^{*}$ are compact at the same time [7, Chapter 10].

Theorem A.2 Suppose $T$ is compact. Then (A.3) has a nonzero solution $u_{n}$ only in the case of $\lambda=\lambda_{n}, n=0,1,2, \ldots$, i.e.

$$
\mathcal{L} u_{n}=\lambda_{n} T u_{n}, \quad n=1,2, \ldots
$$

The system $u_{n}$ forms an orthogonal basis in $W$. The sequence $\lambda_{n}$ forms a nondecreasing sequence of positive numbers,

$$
0<\lambda_{0} \leq \lambda_{1} \leq \lambda_{2} \leq \cdots
$$

and $\lim \lambda_{n}=\infty$.

Remark A.1 The minimal eigenvalue $\lambda_{0}$ satisfies the equality (A.4).

\section{A.2 General case}

Let

$$
\langle u, v\rangle=[u, v]-Q(u, v)
$$

be a symmetric bilinear form, $u, v \in W$. Assume that $Q$ is bounded in both arguments. Moreover, suppose that this form has the representation $Q(u, v)=(Q u, T v)$, where $Q: W \rightarrow H$ is bounded. ${ }^{e}$ Then the equation $[u, v]-(Q u, T v)=(f, T v), \forall v \in W$, is equivalent to

$$
u-T^{*} Q u=T^{*} f
$$

and in the set $D_{\mathcal{L}_{0}}=T^{*}(H)$ to

$$
\mathcal{L} u=\mathcal{L}_{0} u-Q u=f,
$$

where $\mathcal{L}_{0}=\left(T^{*}\right)^{-1}$. 
Theorem A.3 Suppose $T$ is compact. Then the equation

$$
\mathcal{L} u=\mathcal{L}_{0} u-Q u=\lambda T u
$$

has a nonzero solution $u_{n}$ only in the case of $\lambda=\lambda_{n}, n=0,1,2, \ldots$, i.e.

$$
\mathcal{L} u_{n}=\lambda_{n} T u_{n}, \quad n=0,1,2, \ldots
$$

The system $u_{n}$ can be chosen to form an orthogonal basis in the space $W$.

If the form $\langle u, v\rangle=[u, v]-(Q u, T v)$ is lower semi-bounded, i.e.

$$
\inf _{u \neq 0} \frac{\langle u, u\rangle}{(T u, T u)}>-\infty
$$

then spectrum of $\mathcal{L}$ is semi-bounded, and

$$
\lambda_{0}=\inf _{u \neq 0} \frac{(\mathcal{L} u, T u)}{(T u, T u)}=\inf _{u \neq 0} \frac{\langle u, u\rangle}{(T u, T u)}
$$

is the greatest lower bound of the spectrum [7, Chapter 6]. Thus we have the following.

Theorem A.4 If (A.9) holds and $T$ is compact then the eigenvalues $\lambda_{n}$ have a minimum and can be put in increasing order

$$
\lambda_{0} \leq \lambda_{1} \leq \lambda_{2} \leq \cdots
$$

\section{A.3 Positive definiteness and spectral radius of $Q T^{*}$}

Let $\rho=\rho\left(Q T^{*}\right)$ be the spectral radius of the operator $Q T^{*}$. Note that the two operators $Q T^{*}$ and $T^{*} Q$ have the same spectral radius. So

$$
\rho=\sup _{u \neq 0} \frac{\left[T^{*} Q u, u\right]}{[u, u]}=\sup _{u \neq 0} \frac{(Q u, T u)}{[u, u]} .
$$

Lemma A.2 The quadratic form $\langle u, u\rangle$ is positive definite if and only if $\rho<1$.

Proof From (A.11) it follows that $\rho[u, u]-(Q u, T u) \geq 0$ and $\langle u, u\rangle=[u, u]-(Q u, T u) \geq$ $(1-\rho)[u, u]$. If $\rho<1$ then $\langle u, u\rangle$ is positive definite. Conversely from the inequality $[u, u]-$ $(Q u, T u) \geq \varepsilon[u, u](\varepsilon>0)$ it follows that $\rho \leq 1-\varepsilon$.

So if $\rho<1$, then by (A.10)

$$
\lambda_{0} \geq \inf _{u \neq 0} \frac{[u, u]-\rho[u, u]}{(T u, T u)}>0 .
$$

Conversely, suppose $\lambda_{0}>0$. Then

$$
[u, u]-(Q u, T u) \geq \lambda_{0}(T u, T u)>0,
$$


and $\rho \leq 1$. If $(Q u, T u)$ is nonnegative, then spectrum of $T^{*} Q$ is in the segment $[0, \rho]$, and $\rho$ is a point of spectrum. In this case from $\lambda_{0}>0$ it follows that $\rho<1$. So, we have the following.

Theorem A.5 Suppose ( $Q u, T u)$ is nonnegative. The following assertions are equivalent:

1. $\langle u, u\rangle$ is positive definite,

2. $\lambda_{0}>0$,

3. $\rho\left(Q T^{*}\right)<1$.

\section{Appendix 2: A generalization of the Fubini theorem}

We used a change of integration order in an integral, which does not follow from the classic Fubini theorem. The following assertion is taken from the monograph [8]. Note that it was used without proof in [2-4].

Lemma B.1 Let $(X, \mathcal{A})$ and $(Y, \mathcal{B})$ be measurable spaces, $\mu$ be a measure ${ }^{\mathrm{f}}$ on $(X, \mathcal{A}), K: X \times$ $\mathcal{B} \rightarrow[0, \infty]$ be kernel (i.e. for $\mu$-almost all $x \in X, K(x, \cdot)$ is a measure on $(Y, \mathcal{B}), \forall B \in \mathcal{B}$, $K(\cdot, B)$ is $\mu$-measurable on $X)$. Then

1. the function $v$ defined on $\mathcal{A} \times \mathcal{B}$ by the equality

$$
\nu(E)=\int_{X} K\left(x, E_{x}\right) \mu(d x), \quad \text { where } E_{x}=\{y:(x, y) \in E\},
$$

is measure;

2. iff $: X \times Y \rightarrow[-\infty, \infty]$ is v-measurable on $X \times Y$, then

$$
\int_{X \times Y} f(x, y) d \nu=\int_{X}\left(\int_{Y} f(x, y) K(x, d y)\right) \mu(d x) .
$$

Remark B.1 The function $v$ is the Lebesgue extension from the set of rectangles

$$
v(A \times B)=\int_{A} K(x, B) \mu(d x), \quad A \in \mathcal{A}, B \in \mathcal{B} .
$$

'Department of Mathematics, Moscow State University of Economics, Statistics and Informatics, Nezhinskaya 7, Moscow, 119501, Russia. ${ }^{2}$ Instituto Superior de Transportes e Comunicações, Prolong. da Av. Kim II Sung (IFT/TDM) - Edifício D1, Maputo, 2088, Mozambique. 


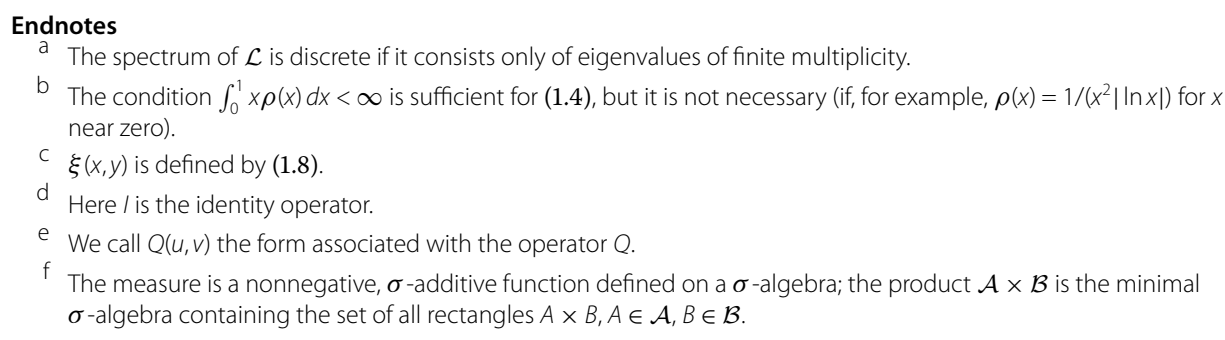

Received: 5 February 2014 Accepted: 4 April 2014 Published: 07 May 2014

\section{References}

1. Glazman, IM: Direct Methods of Qualitative Spectral Analysis of Singular Differential Operators. Israel Program for Scientific Translation, Jerusalem (1965)

2. Labovskij, S: On the Sturm-Liouville problem for a linear singular functional-differential equation. Russ. Math. 40(11), 50-56 (1996) (Russian original: Izv. Vysš. Učebn. Zaved., Mat. 11(414), 48-53 (1996)). Zbl 0909.34070

3. Labovskii, S: Little vibrations of an abstract mechanical system and corresponding eigenvalue problem. Funct. Differ. Equ. 6(1-2), 155-167 (1999). Zbl 1041.34050

4. Labovskiy, S: Small vibrations of mechanical systems. Funct. Differ. Equ. 16(3), 447-468 (2009). Zbl 05708058

5. Kantorovich, LV, Akilov, GP: Functional Analysis. Nauka, Moscow (1984) (Russian)

6. Akhiezer, NI, Glazman, IM: Theory of Linear Operators in Hilbert Space. Dover, New York (1993). Zbl 0874.47001

7. Birman, MS, Solomjak, MZ: Spectral Theory of Self-Adjoint Operators in Hilbert Space. Reidel, Dordrecht (1987)

8. Cohn, DL: Measure Theory. Birkhäuser, Boston (1993). Zbl 0860.28001

10.1186/1687-2770-2014-102

Cite this article as: Labovskiy and Getimane: On discreteness of spectrum and positivity of the Green's function for a second order functional-differential operator on semiaxis. Boundary Value Problems 2014, 2014:102

\section{Submit your manuscript to a SpringerOpen ${ }^{\circ}$ journal and benefit from:}

- Convenient online submission

Rigorous peer review

- Immediate publication on acceptance

- Open access: articles freely available online

- High visibility within the field

- Retaining the copyright to your article 